\title{
Machine Learning Assisted Signal Detection in Ambient Backscatter Communication Networks
}

This paper was downloaded from TechRxiv (https://www.techrxiv.org).

LICENSE

CC BY 4.0

SUBMISSION DATE / POSTED DATE

28-10-2021 / 01-11-2021

CITATION

Toro, Usman Saleh; Elhalawany, Basem M.; Wong, Aslan B; Wang, Lu; Wu, Kaishun (2021): Machine Learning Assisted Signal Detection in Ambient Backscatter Communication Networks. TechRxiv. Preprint. https://doi.org/10.36227/techrxiv.16895809.v1

$\mathrm{DOI}$

10.36227/techrxiv.16895809.v1 


\title{
Machine Learning Assisted Signal Detection in Ambient Backscatter Communication Networks
}

\author{
Usman Saleh Toro, Graduate Student Member, IEEE, Basem M. ElHalawany, Senior Member, IEEE, \\ Aslan B. Wong, Graduate Student Member, IEEE, Lu Wang, Senior Member, IEEE, and Kaishun Wu, Senior \\ Member, IEEE
}

\begin{abstract}
Ambient backscatter communication (AmBC) has emerged as a promising paradigm for enabling sustainable lowpower operation of Internet of Things (IoT) devices. This is due to its ability to enable sensing and communication through backscattering ambient wireless signals (such as WiFi and TV signals). But a great impediment to AmBC-enabled networks is the difficulty in decoding the backscatter signals because the ambient signals are usually modulated and meant for other legacy receivers rather than $\mathrm{AmBC}$ devices. Drawing from the ability of machine learning (ML) to enhance the performance of wireless communication systems, some ML-aided techniques have been developed to assist signal detection in AmBC. Hence, this paper aims to provide a comprehensive overview of the subject by; describing the operation of the AmBC network, highlighting the major challenges to signal detection in $\mathrm{AmBC}$, discussing and comparing the performance of some existing ML-assisted solutions to AmBC signal detection, and highlighting some future research that could be carried out on the subject.
\end{abstract}

Index Terms-machine learning, signal detection, ambient backscatter communication, communication networks.

\section{INTRODUCTION}

$\mathbf{I}$ NTERNET of Things (IoT) will drastically revolutionize human-to-human $(\mathrm{H} 2 \mathrm{H})$, human-to-device (H2D), and machine-to-machine (M2M) interactions in the nearest future. This will lead to myriads of intelligent pervasive applications in various sectors of human life including agriculture, transportation, commerce, and healthcare [1].The key to enabling such applications is addressing the limited energy problem of IoT devices. Recently, ambient backscatter communication $(\mathrm{AmBC})$ has emerged as a strong contender for enabling lowpower and sustainable operation of IoT devices.

Backscatter communication (BackCom) involves the transmission of sensed data at a backscatter device (BD) or tag by modulating it unto an incident signal from a source (or reader) and reflecting the modulated incident signal to a receiver. This allows the BD to sense data and transmit signals without actively generating a carrier signal. Thereby, providing a very low-powered means of sensing and communication and also enabling the development of battery-free sensors. To enable battery-free operation, the BD harvests energy from the incident signal and stores it in capacitors for powering the BD's operation. To enhance the sustainable operation of the BDs,

All authors are with College of Computer Science and Software Engineering, Shenzhen University, Shenzhen 518060, China. Lu Wang is the corresponding author (email: \{torousmansaleh2019@email.szu.edu.cn, basem.mamdoh@szu.edu.cn, aslan@szu.edu.cn, wanglu@szu.edu.cn, wu@szu.edu.cn\}). Basem is also associated with Benha University, Egypt. energy harvesting (EH) techniques [2] have been developed to supplement the harvested from the incident ambient signal. In addition to the benefits of traditional BackCom, AmBC shown in Fig. 1 leverages ambient wireless signals (such as WiFi, GSM, TV, and frequency modulated (FM) radio) as an incident signal at the BD for modulating sensed data. This eliminates the need for deploying a dedicated source for carrier generation and further decreases the cost of deploying sensors in the IoT ecosystem [2].

Despite the benefits that come along with deploying AmBC in wireless networks, decoding the backscatter signal at the receiver is challenging due to various reasons. They include interference at the receiver from direct path signal [3], challenges in backscatter channel estimation [3], low strength of backscatter signal at the receiver [4], absence of distributed multiple access (MAC) protocol [4], and complex operation dynamics of AmBC network components. Techniques to address these challenges have been developed but further work to minimize the challenges is still required. Hence, considering the ability of machine learning (ML) techniques to leverage trained models for improving wireless communication systems [5], several works have developed ML-based solutions to address the problem of signal detection in the AmBC network [6]-[13] despite being at their early stages of deployment. To provide a comprehensive view of the subject, this paper aims to bring a survey of the existing ML-based solutions and also provides a discussion of future research on the subject.

The paper is structured as follows: Section I gives an introduction to ML-assisted signal detection in AmBC. The operation of AmBC is described in section II. Then, section III describes the bottlenecks to signal detection in the AmBC network. Some existing ML-assisted signal detection solutions are discussed in section IV. Further, a comparison of the performance of existing techniques in assisting signal detection in $\mathrm{AmBC}$ is presented in V. Section VI describes some future research that could further enhance ML-assisted signal detection. Lastly, section VII concludes the paper.

\section{Overview ON AMBC NETWORK OPERATION}

This section explains the operation principle of an AmBC network.

The AmBC network consists of an ambient source, a BD, and a receiver as shown in Fig. 1(a). At the top level, AmBC operates just like a heliograph. The ambient source transmits signals which are meant for other legacy devices such as 


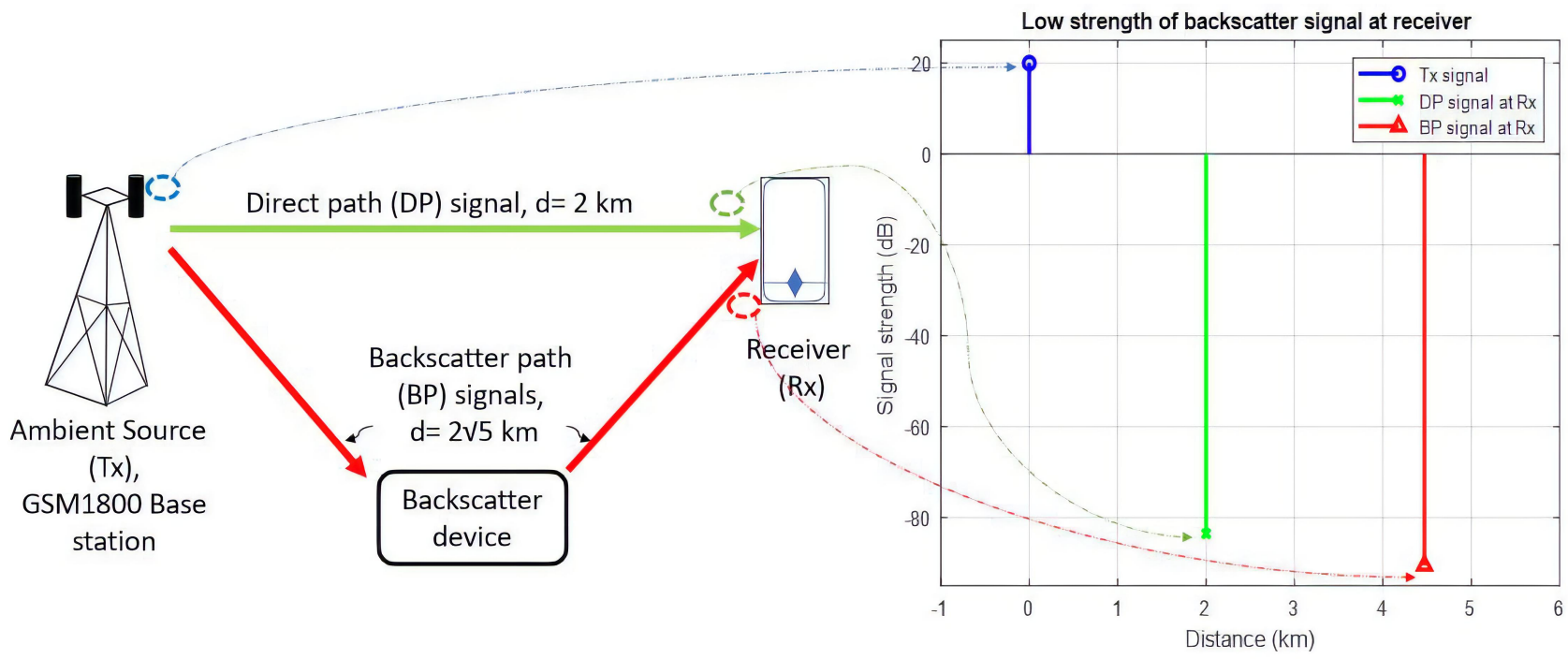

(a) AmBC network model with GSM1800 Base station as a source

(b) Strengths of the signals in (a) under free space loss

Fig. 1: AmBC system model showing signal strengths at various positions with free space loss.

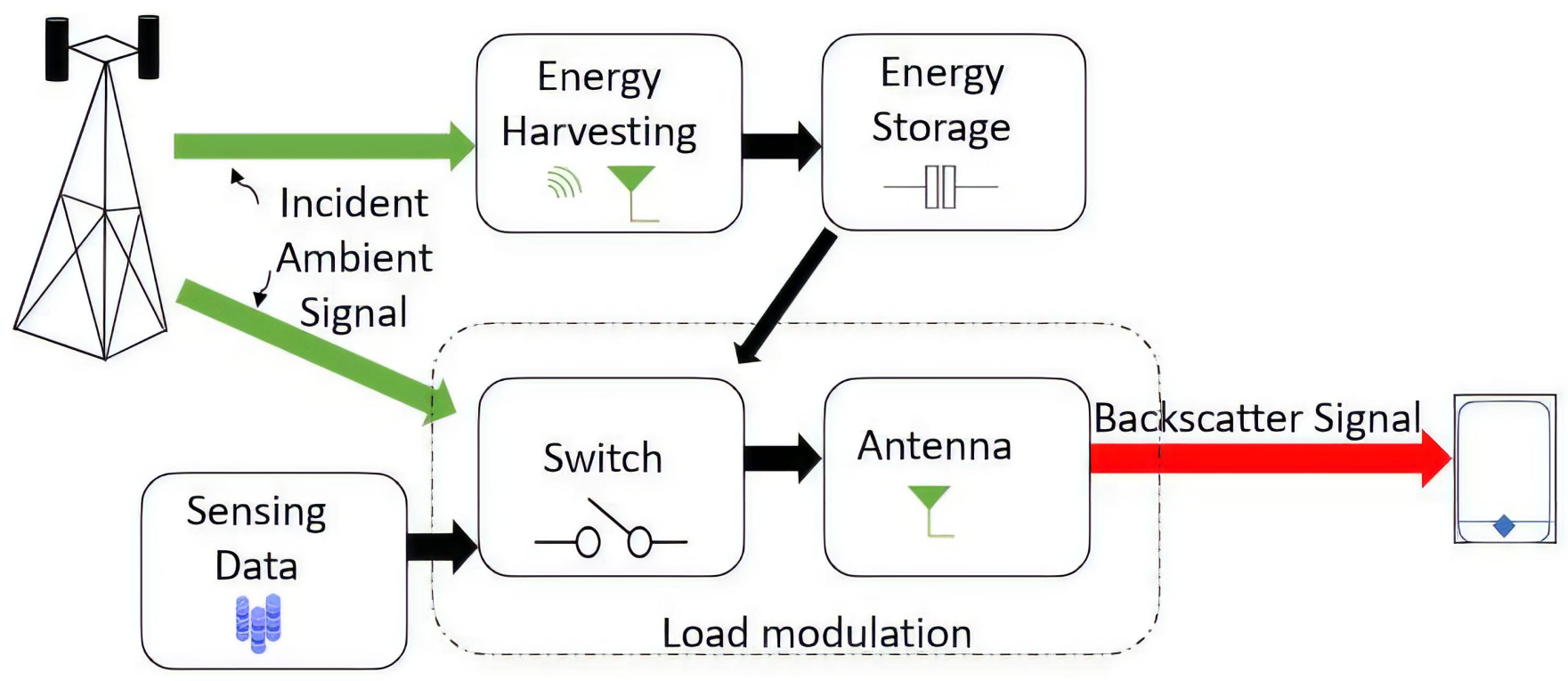

Fig. 2: Operation of AmBC

mobile phones, TV, and FM radio. When the ambient signals are incident onto the $\mathrm{BD}$, they provide two main functions. They are: serve as an energy source and serve as a carrier signal for modulating sensing data. As an energy source, the ambient signals will be converted into electrical energy and stored in capacitors or small batteries. And for modulating sensing data, a technique called load modulation is leveraged to enable sensing data at the BD to be reflected towards a receiver. Load modulation involves switching the impedance of the BD between various states based on the value of data to be transmitted by the BD. Since the weak incident signals at the BD are on the same channel as the received signals at legacy devices, the BD could apply some techniques that can move the backscatter signal to a different channel to avoid co- channel interference. Further, the BD could amplify the signal strength of the reflected signal to lessen the impact of fading on its path to the receiver. At the receiver, the backscatter signal is decoded to extract the sensing data. Since the receiver could be a device with high computational resources such as mobile phones and computers, they allow signal processing and machine learning techniques to be adopted by the receiver for enhanced signal detection.

\section{Bottlenecks to SignAl DeteCtion in AMBC NETWORK}

This section identifies and describes some of the most critical challenges to signal detection in AmBC networks. The challenges are arranged based on the layer of OSI network they 
affect. These challenges are shown in Fig. 3.

1) Direct path signal interference (Physical layer): As shown in Fig. 1(a), the stronger direct path signal and the weaker backscatter signals are picked up by the same receiver. Since there are transmission channels from the ambient source to $\mathrm{BD}$ and receiver, co-channel interference will occur at the receiver of AmBC systems that don't have techniques to combat such type of interference. Though some BDs can shift the backscatter signal to another channel, techniques to nullify or avoid the stronger direct path signals are required for efficient signal detection.

2) Low strength of backscatter signal (Physical layer): Naturally wireless signals will fade over distance. In conventional communication systems, signal strength will degrade with the inverse of squared distance. But since AmBC consists of the incident signal and backscatter signal paths, the doubly-faded backscatter signal at the receiver becomes very weak (sometimes beyond the noise floor) at the receiver. This leads to difficulty in signal detection at the receiver.

3) Backscatter channel estimation (Physical layer): Channel estimation at the receiver is a vital process in wireless communication for ensuring accurate decoding of the received signal. For that, the receiver needs to have access to the channel via which data is transmitted. In the AmBC system shown in Fig. 1(a), the receiver does not have access to the channel between the ambient source and BD. This results in challenges in estimating the backscatter channel and hence signal detection.

4) Absence of MAC protocol (MAC layer): Since the ambient sources in AmBC are meant for other legacy devices, they do not provide MAC for BDs. Further, the AmBC network could consist of multiple BDs that leverage the same ambient source for sensing and communication. In such scenarios, the tags could transmit data to the receiver in a multi-hop or single-hop manner. In single-hop transmission, the BDs could transmit at the same time slots leading to collisions at the receiver. While in multi-hop transmission, the order of packet transmission between tags could be distorted thereby preventing correct data from reaching the receiver.

5) Dynamics of AmBC network components (Network layer): The benefits of eliminating a dedicated source in AmBC come at the expense of having to consider the operation of the ambient source when designing signal detection techniques. This is more complicated when the signal source is a smart interferer whose operation is difficult to predict. Hence, an efficient signal detection technique in $\mathrm{AmBC}$ requires taking cognizance of the dynamic operations of both ambient sources and BDs.

\section{Existing Solutions}

In this section, we shed light on several attempts to address the bottlenecks of signal detection in an AmBC network using various ML-based techniques. The solutions are discussed based on the category of underlying ML algorithms adopted. Also, a comparison of the solutions is shown in Table I.

\section{A. Unsupervised Learning (UL):}

In order to develop a technique for detecting reflected ambient signals without the need for channel estimation, authors in [6] leveraged the clustering phenomenon. The clustering phenomenon in the AmBC system network allows reflected signals from tags (or BD) to naturally fall into clusters at the receiver. The clusters are formed based on the type of information contained in the reflected signal. Hence, the authors developed an AmBC network that adopts amplitude modulation at the tag. The amplitude modulation adopted allows the tag to assign two distinct energy levels to the reflected signal which will eventually form two clusters at the receiver. Then, UL algorithms based on expectation maximization (EM) were used to classify and detect the transmitted data from the tag. In order to optimize the detection thresholds, learned parameters from the developed algorithms were used to obtain a minimum bit error rate (BER).

Similarly, the author in [7] leveraged UL to aid signal detection in AmBC. Rather than exploiting the clustering phenomenon of AmBC, joint coherent signal estimation and detection at the receiver were leveraged. The AmBC network was modeled with an orthogonal frequency division multiplexing (OFDM) enabled source, a BD, and a receiver. Since coherent backscatter signal detection requires the channel state information (CSI) of the backscatter device. A matched-filter was used at the backscatter device to estimate the carrier CSI from the source. The estimated carrier CSI is then used at the receiver to efficiently estimate the backscatter signal. Due to the computational complexity of decoding at the receiver, an EM-based algorithm with complexity linear to the length of OFDM was developed to aid the detection of backscatter signals.

UL learns patterns from unlabeled data and thus is suitable for signal detection in AmBC without channel estimation. Yet if the wireless environment becomes complicated, there is little room for improvement. To achieve a high signal detection accuracy, traditional signal processing techniques, such as de-noising and signal estimation are required to better estimate and detect the ambient signals. With advanced signal processing techniques, the detection efficiency and accuracy can be much improved. 


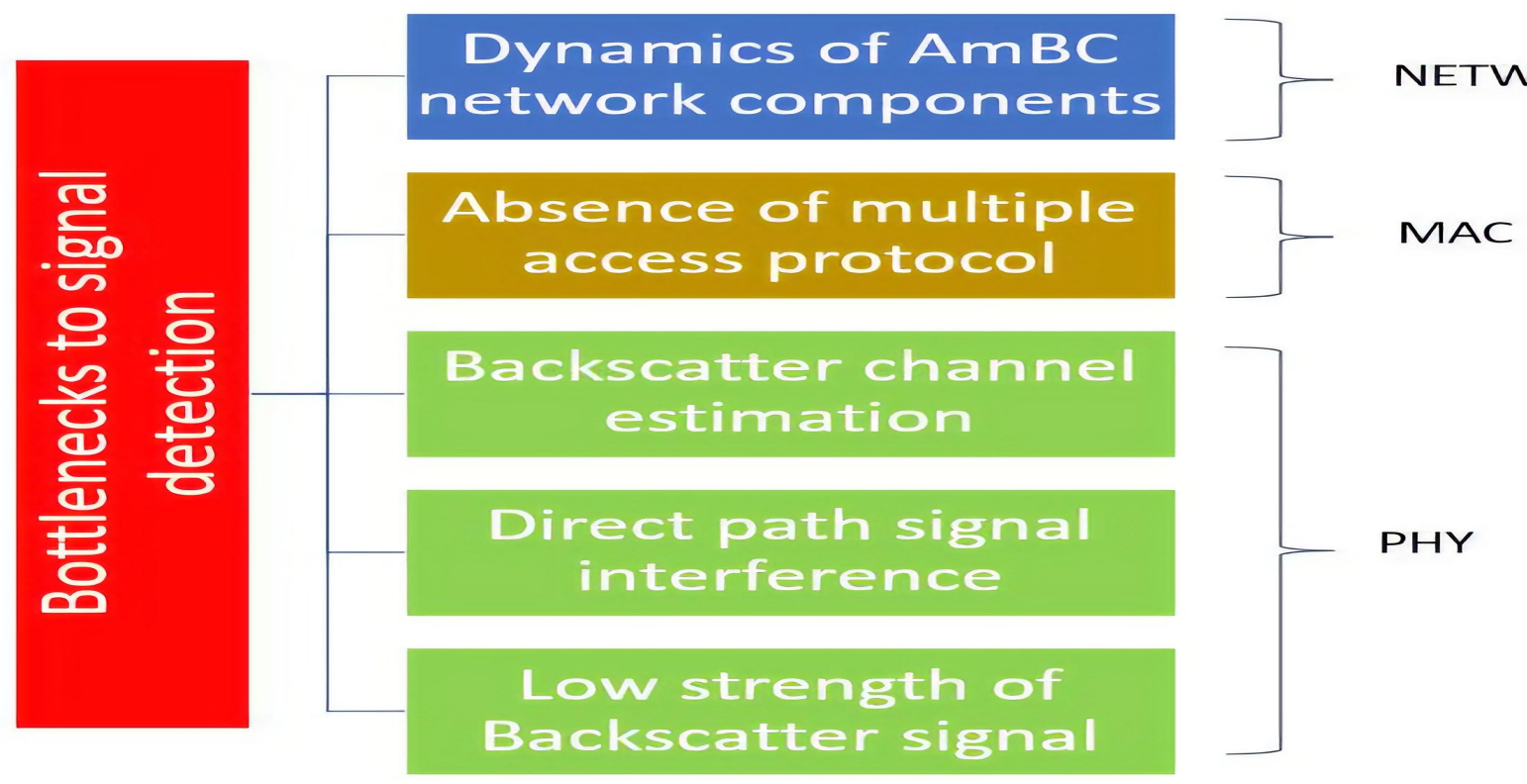

Fig. 3: Bottlenecks to signal detection in AmBC network.

TABLE I: Comparison of existing solutions

\begin{tabular}{|c|c|c|c|}
\hline \hline Reference & ML category & Phenomenon employed to aid signal detection & Main bottleneck(s) addressed \\
\hline \hline$[6]$ & UL & Clustering of received signals & Backscatter channel estimation \\
\hline$[7]$ & UL & Joint coherent signal detection and channel estimation & Backscatter channel estimation \\
\hline$[8]$ & SL & $\begin{array}{c}\text { Varying energy levels of received data } \\
\text { Dow strengath signal interference, } \\
\text { stref backscatter signal }\end{array}$ \\
\hline$[9]$ & SL & Label-assisted clustering of received signal & $\begin{array}{c}\text { Backscatter channel estimation } \\
\text { Direct-path signal interference, } \\
\text { Low strength of backscatter signal }\end{array}$ \\
\hline$[10]$ & RL & An SDN-based AmBC network & Dynamics of AmBC network components \\
\hline$[11]$ & RL & Feature extraction and transfer learning & Backscatter channel estimation \\
\hline$[12]$ & DL & Feature extraction and residual learning & Backscatter channel estimation \\
\hline$[13]$ & DL & &
\end{tabular}

TABLE II: Performance comparison of signal detection techniques

\begin{tabular}{|c|c|c|c|}
\hline \hline Reference & Detection technique & Model properties & Result (BER) \\
\hline \hline Simulated & signal processing & $\begin{array}{c}\text { Ambient RF source, Modulation-FM, } \\
\text { Carrier frequency- 90 MHz, SNR:10 dB }\end{array}$ & 0.6536 \\
& UL & Ambient RF source, Modulation-QPSK, SNR:10 dB & 0.04 \\
\hline$[6]$ & SL & Ambient RF source, Modulation-16QAM, SNR:10 dB & 0.007 \\
\hline$[9]$ & DL & Ambient RF source, Modulation-QPSK, SNR:10 dB & 0.0008 \\
\hline$[12]$ & \multicolumn{2}{|l}{} \\
\hline
\end{tabular}

\section{B. Supervised Learning (SL):}

Considering tag signal detection as a classification task, authors in [8] deployed SL algorithms (support vector machines and random forest) for backscatter tag signal detection. Simple conventional signal detectors in AmBC use energy detection to identify transmitted tag symbols (0 and 1) which signify backscatter and non-backscatter states. But the energy detection techniques suffer due to the low signal-to-noise ratio (SNR) of backscatter path signals. Hence, the authors used the received signals and tag symbols as training data and labels respectively. The tag first transmits some labeled symbols for training at the receiver. Then, the trained model is used to detect tag data by classifying the received data into two groups which correspond to 0 and 1 . The proposed SL-based detection showed better performance (low BER) in low SNR conditions over conventional energy detection-based tag signal detection.

Leveraging the clustering phenomenon from a different perspective, authors in [9] developed an SL-like technique to aid the detection of backscatter signals without the need for channel estimation. In the adopted model, a multi-antenna type receiver was deployed to decode backscatter signals from a modulated RF source through a tag. Since decoding clustered received data is tasking, the authors proposed a label-assisted clustering technique. In the proposed technique, the tag assigns known labels before each data transmission. Hence, the received data will fall into clusters based on the known labels. An EM algorithm with less complexity due to modulation constraints from the tag and RF source was used to learn parameters which are then used to decode the transmitted signal. This technique achieved similar results to 
an optimal detector with perfect CSI.

SL algorithms are commonly used for classification, regression, and recognition tasks. Thus, it is effective to address signal interferences and estimate wireless channels. Yet it requires data training in advance, and the model training is highly dependent on the wireless environment. SL itself has not much room to be improved when applied to another environment. With the help of Transfer learning (TL), training knowledge can be stored and applied to a different wireless environment for interference cancellation and channel estimation.

\section{Reinforcement Learning (RL):}

An AmBC network contains multiple (AmBC and legacy) devices co-existing in a heterogeneous nature making the interaction between those devices difficult due to interference. In order to address this problem, authors in [10] proposed a software-defined network (SDN) based AmBC network. But the SDN-based AmBC network needs to learn from the operating environment in order to manage interference. Hence, an RL technique was developed to achieve optimal rewards. The RL technique enabled efficient interference management by controlling the transmit power levels of heterogeneous devices. Results of the SDN-based AmBC showed improved performance among legacy devices and ensured the required quality of service (QoS) for AmBC devices.

In order to explore the ability to combine RL and game theory for avoiding interference, authors in [11] developed a technique for detecting backscatter signals in the presence of a smart interferer. In order to achieve that, the interaction between an interferer and the AmBC system was modeled as a game. Since the AmBC device's operation time is divided into energy harvesting time (EHT) and backscatter time (BT), those time durations were considered when developing the utility functions. For each sub-game, RL was used to obtain the optimal policy for the game due to the non-availability of the interferer operation times. The developed technique showed better signal detection when compared with techniques that consider random and fixed BTs.

RL has the ability to interact with the environment, thus it is suitable to fight against dynamics of network components and detect AmBC signals with the presence of interference. However, if the network is complex with large-scale size, the state and action spaces are usually large in AmBC networks, and RL may not be able to find the optimal policy in a reasonable time and thus deteriorate the performance. Possible solutions may involve DL, known as Deep Reinforcement Learning (DRL) to deal with such scenarios.

\section{Deep Learning (DL):}

As a novel technique with a strong ability to extract features from data, authors in [12] developed a deep transfer learning (DTL) framework. DTL involves adopting a deep neural network (DNN) to extract the features of the received signal at the tag without the need for CSI. But in order to capture the time-varying conditions of the $\mathrm{AmBC}$ channel and avoid the need for large training data required to achieve low BER, the authors adopted; offline learning, transfer learning, and online tag data detection. Hence, the tag parameters that were learned during the offline tag detection are transferred for use in detecting the parameters of other related tags in real-time. Simulation results of the proposed framework showed near-optimal performance when compared with perfect CSI conditions.

On the other hand, authors in [13] leveraged deep residual learning to estimate CSI and enhance signal detection in AmBC. Since channel estimation in AmBC is challenging, the authors modeled it as a denoising problem. In the model, $\mathrm{CNN}$ was used to directly recover the channel coefficients (in time and frequency domains) from the received pilot signals at the receiver. Simulated results of the proposed model showed similar results to systems with optimal minimum mean square error (MMSE) estimator.

As DL has a strong ability to extract features, it is feasible to apply DL for channel estimation with a complex AmBC environment. DL has the potential to achieve high estimation accuracy. The only problem is that it requires a very large amount of data, and it is extremely expensive to train due to complex data models. Therefore, reducing the computational expense is indispensable for AmBC networks.

\section{Case Study: Conventional versus ML-Assisted DETECTION}

In this section, we compare the performance of a conventional signal detection technique with some ML-assisted signal detection techniques of $\mathrm{AmBC}$ models. The conventional signal detection technique considered is the rule-based signal processing of an FM radio demodulator, while the considered ML-assisted techniques are the UL [6], SL [9], and DL [12]. To efficiently characterize the performance, we consider the type of ambient source, the modulation at the ambient source and the SNR at the receiver as the AmBC model parameters.

The conventional model considered in this section is an AmBC system that exploits ambient FM radio signals as the source of excitation. The system is modelled and developed using the communication toolbox of Simulink. The FM message signal is modeled as a sequence of randomly generated bits, while a carrier signal of $90 \mathrm{MHz}$ is generated at the transmitter. The modulated FM signal is transmitted over a fading channel to the $\mathrm{BD}$, then to the receiver. At the receiver, we set the SNR to $10 \mathrm{~dB}$ in order to observe the demodulated results.

The Bit Error Ratio (BER) is used as the performance metric in the experiment. For the conventional system, the original transmitted bits (message signal) were compared to the decoded bits at the FM demodulator to obtain the BER of the system. For the ML-assisted techniques ( [6], [9] and 
[12]), we obtained the results (BER) of those systems where the AmBC systems designed adopted signal detection schemes at the receiver with an SNR of $10 \mathrm{~dB}$ and fading ambient signals.

The FM radio-based AmBC simulation parameters and the results of existing solutions are shown in Table II. The results show that the conventional detection method of the FM radiobased AmBC has the highest BER compared to other MLassisted techniques. This is because the demodulator adopts rule-based (signal processing) techniques to detect the transmitted signal. But the BER can be improved when advanced signal processing techniques are adopted. We also observe that the BER values show significant improvements with the adoption of ML techniques at the receiver. Furthermore, the DL method shows the best performance over the other ML techniques due to its strong feature extraction ability when properly modelled according to the application scenario. Hence, we conclude that ML-assisted signal detection improves the performance of AmBC networks.

\section{FUTURE WORK}

This section discusses some open research issues related to signal detection in the AmBC network and possible suggestions on how to address those issues.

1) Accuracy vs Energy trade-off: While improving the accuracy of ML techniques for backscatter signal detection is desired, the limited power budget of AmBC devices is still a bottleneck to BDs. This becomes more evident when a wearable device is used as a receiver for backscatter signal detection. In such a scenario, leveraging an ML-assisted technique for signal detection requires training a model deployed on the wearable device with some amount of dataset. And the higher the amount of training data, the more accurate the model will perform while increasing the power consumption of the already power-stretched wearable device. Hence considering both ends of the situation, future ML-assisted techniques need to deploy low training data in order to achieve appreciable detection accuracy without overstretching the power demands of AmBC receivers.

2) Intelligent non-coherent techniques: Non-coherent signal detection techniques have shown promising results when applied in detecting AmBC signals despite their nondependence on channel estimation. But despite that, there is still a need to develop ML-assisted techniques that can capture the complexities of the AmBC network. From the properties of the channels (incident path and backscatter path) of AmBC, dynamics of incident signal from an ambient source, the interaction between components (ambient source, backscatter device, and interferer) of the AmBC network to the operation of the backscatter tags itself. Incorporating these parameters in designing non-coherent signal detection techniques will further enhance its appeal for adoption in AmBC networks. Pioneering work by authors in [14] has shown the promise of incorporating channel properties (time-selective fading) in improving signal detection.

3) Intelligent re-configurable architecture of receiver: The existing ML-assisted techniques involve the development and training of models which inherently affect the software architecture of the AmBC receiver. In other words, the hardware architecture of the AmBC receiver does not incorporate some elements of intelligent reconfigurability. Developing hardware architectures at the receiver that can optimize the signal detection process at the receiver due to: available energy, modulation adopted by the backscatter tag, and CSI will certainly enhance the overall detection process at the receiver. For instance, AmBC systems have shown the promise of enhanced signal detection when multiple tag antennas are adopted [15]. With many ambient sources (such as WiFi access point (AP) and cellular base stations) adopting OFDM, the benefits of channel diversity can be fully leveraged in AmBC signal detection by adopting multiple antennas at the source, tag, and receiver of an AmBC network to achieve higher throughput and lower BER.

\section{CONCLUSION}

Addressing the signal detection problem of AmBC will go a long way in enhancing its appeal for enabling sustainable communication networks. The comprehensive discussion presented in this paper addressed the contributors to difficulty in signal detection and explicitly describes how existing solutions leveraged ML to assist signal detection. Lastly, the future research issues discussed provides possible succinct ways of improving ML-assisted signal detection. The results in our case study show that DL techniques have shown great improvements in solving the signal detection problem. However, to characterize how well the different sub-categories of DL models solves the problem in different AmBC models needs further investigations, which can be pursued in future work.

\section{ACKNOWLEDGMENT}

This research was supported in part by the china NSFC 61872246, Guangdong Special Support Program, Lu WANG is the corresponding author.

\section{REFERENCES}

[1] F. Jameel, R. Duan, Z. Chang, A. Liljemark, T. Ristaniemi, and R. Jantti, "Applications of backscatter communications for healthcare networks," IEEE Network, vol. 33, no. 6, pp. 50-57, 2019.

[2] N. Van Huynh, D. T. Hoang, X. Lu, D. Niyato, P. Wang, and D. I. Kim, "Ambient backscatter communications: A contemporary survey," IEEE Comm. Surveys Tut., vol. 20, no. 4, pp. 2889-2922, 2018.

[3] D. Darsena, G. Gelli, and F. Verde, "Joint channel estimation, interference cancellation, and data detection for ambient backscatter communications," in Proc. SPAWC. IEEE, 2018, pp. 1-5.

[4] G. Wang, F. Gao, R. Fan, and C. Tellambura, "Ambient backscatter communication systems: Detection and performance analysis," IEEE Trans. Comm., vol. 64, no. 11, pp. 4836-4846, 2016. 
[5] Y. Sun, M. Peng, Y. Zhou, Y. Huang, and S. Mao, "Application of machine learning in wireless networks: Key techniques and open issues," IEEE Comm. Surveys Tut., vol. 21, no. 4, pp. 3072-3108, 2019.

[6] Q. Zhang and Y.-C. Liang, "Signal detection for ambient backscatter communications using unsupervised learning," in Proc. IEEE Globecom Workshops. IEEE, 2017, pp. 1-6.

[7] J. Choi, "Matched-filter-based backscatter communication for iot devices over ambient ofdm carrier," IEEE Internet of Things Journal, vol. 6, no. 6, pp. 10229-10239, 2019

[8] Y. Hu, P. Wang, Z. Lin, M. Ding, and Y.-C. Liang, "Machine learning based signal detection for ambient backscatter communications," in Proc. ICC. IEEE, 2019, pp. 1-6.

[9] Q. Zhang, H. Guo, Y.-C. Liang, and X. Yuan, "Constellation learningbased signal detection for ambient backscatter communication systems," IEEE Journal on Selected Areas in Communications, vol. 37, no. 2, pp. 452-463, 2018.

[10] F. Jameel, W. U. Khan, M. A. Jamshed, H. Pervaiz, Q. Abbasi, and R. Jäntti, "Reinforcement learning for scalable and reliable power allocation in sdn-based backscatter heterogeneous network," in Proc. IEEE INFOCOM Workshops. IEEE, 2020, pp. 1069-1074.

[11] A. Rahmati and H. Dai, "Reinforcement learning for interference avoidance game in rf-powered backscatter communications," in Proc. IEEE ICC . IEEE, 2019, pp. 1-6.

[12] C. Liu, Z. Wei, D. W. K. Ng, J. Yuan, and Y.-C. Liang, "Deep transfer learning for signal detection in ambient backscatter communications," IEEE Trans. Wireless Comm., vol. 20, no. 3, pp. 1624-1638, 2021.

[13] X. Liu, C. Liu, Y. Li, B. Vucetic, and D. W. K. Ng, "Deep residual learning-assisted channel estimation in ambient backscatter communications," IEEE Wireless Comm. Lett., vol. 10, no. 2, pp. 339-343, 2020

[14] J. K. Devineni and H. S. Dhillon, "Non-coherent detection and bit error rate for an ambient backscatter link in time-selective fading," IEEE Trans. Comm., vol. 69, no. 1, pp. 602-618, 2021.

[15] C. Chen, G. Wang, P. D. Diamantoulakis, R. He, G. K. Karagiannidis, and C. Tellambura, "Signal detection and optimal antenna selection for ambient backscatter communications with multi-antenna tags," IEEE Trans. Comm., vol. 68, no. 1, pp. 466-479, 2019.

Usman Saleh Toro is a $\mathrm{PhD}$ student at the College of Computer Science and Software Engineering at Shenzhen University, China. He obtained MSc (2014) and BEng (2011) from University of Nottingham and University of Bradford Respectively. Currently, $\mathrm{He}$ is carrying out his $\mathrm{PhD}$ studies under the supervision of Distinguished Professor Kaishun Wu. His research is focused on the areas of ultra-low power sensing in IoT, Backscatter Communication and Wireless networks. He is also affiliated to the Department of Computer and Communications Engineering at Abubakar Tafawa Balewa University, Nigeria.
Basem M. ElHalawany received the Master's degree in 2011 from Benha University, Egypt, and Ph.D. in 2014 from Egypt-Japan University of Science and Technology, Egypt. He served as associate research fellow with smart sensing and mobile computing laboratory, Shenzhen university, China and EJUST Center, Kyushu University, Japan. He also holds the position of an associate professor at Faculty of Engineering at Shoubra, Benha University, Egypt. His research interests include performance analysis, resource management, and optimization in wireless networks, NOMA, and machine-learning applications in communication. He has published over 35 high quality research papers in international leading journals and primer conferences.

Aslan Butjamlong Wong is pursuing the $\mathrm{PhD}$ degree at the College of Computer Science and Software Engineering, Shenzhen University. He is supervised by Distinguished Professor Kaishun Wu. His research focuses on human-computer interaction and cognitive science. He is a member of IEEE, ACM, Society of Petroleum Engineers and Engineer Australia.

Lu Wang received the B.S. degree in communication engineering from Nankai University, Tianjin, China, in 2009 and the Ph.D. degree in computer science and engineering from the Hong Kong University of Science and Technology, Hong Kong, in 2013. She is currently an Associate Professor with the College of Computer Science and Software Engineering, Shenzhen University, Shenzhen, China. Her current research interests include wireless communications and mobile computing.

Kaishun Wu received his Ph.D. degree in computer science and engineering from HKUST in 2011. Then, he worked as a research assistant professor at HKUST. In 2013, he joined SZU as a distinguished professor. He has co-authored 2 books and published over 100 high quality research papers in international leading journals and primer conferences, like IEEE TMC, IEEE TPDS, ACM MobiCom, IEEE INFOCOM. He is the inventor of 6 US and over 100 Chinese pending patents. He received 2012 Hong Kong Young Scientist Award, 2014 Hong Kong ICT Awards: Best Innovation and 2014 IEEE ComSoc Asia-Pacific Outstanding Young Researcher Award. 